\title{
Finite-Size Constraints on Nucleation of Hadrons in a Quark-Gluon Plasma
}

\author{
Eduardo S. Fraga \\ Instituto de Física, Universidade Federal do Rio de Janeiro, Cx.P. 68528, Rio de Janeiro, 21941-972, RJ, Brazil \\ and Raju Venugopalan \\ Department of Physics and RIKEN-BNL Research Center, Brookhaven National Laboratory, Upton, NY 11973-5000, USA
}

Received on 15 August, 2003.

\begin{abstract}
We discuss finite-size effects on homogeneous nucleation in first-order phase transitions. We study their implications for cosmological phase transitions and to the hadronization of a quark-gluon plasma generated in high-energy heavy ion collisions.
\end{abstract}

Finite-size scaling has achieved an immense success in the study of equilibrium critical phenomena. On the other hand, systematic studies of finite size effects in the case of metastable decays and other nonequilibrium processes are rare $[1,2]$. In this paper, we discuss finite size effects on the dynamics of homogeneous nucleation in a first-order temperature-driven transition (For more details, see [3]). In particular, we consider the case of cosmological phase transitions in the early universe [4], and that of a quark-gluon plasma (QGP) decay into hadronic matter in a high-energy heavy ion collision $[5,6]$. The former might provide sensible mechanisms to explain the baryon number asymmetry in the universe and primordial nucleosynthesis [7, 8], whereas the latter is expected to be observed [9] at BNL Relativistic Heavy Ion Collider (RHIC). The length and time scales involved in each of these cases differ by several orders of magnitude.

In the usual description of homogeneous nucleation [1], there are two ways in which the finite size of the system can affect the formation and evolution of bubbles and, consequently, the dynamics of phase conversion. Firstly, one has to consider the effects on the nucleation rate and the early stage growth of the bubbles. As will be shown below, this correction comes about through an intrinsic uncertainty in the determination of the supercooling undergone by the system. For the cases considered here, it brings only minor modifications to a description which assumes an infinite system. The second and, in general, most important finite-size effect is its influence on the domain coarsening process, or late-stage growth of the bubbles. The relevant length scales here are the typical size of the system, the radius of the critical bubble and the correlation length.

In a continuum description of a first-order phase transition, it is common to consider a coarse-grained free energy, $F$, of the Landau-Ginzburg form with temperaturedependent coefficients [1]. (In the case of QCD, such a free energy can be obtained, for instance, from the oneloop effective potential of a linear sigma model coupled to quarks $[10,11]$.) The nucleation rate can be expressed as $\Gamma=\mathcal{P} e^{-F_{b} / T}$, where $F_{b}$ is the free energy of a critical bubble, with radius $R_{c}$, and the prefactor $\mathcal{P}$ measures statistical and dynamical fluctuations about the saddle point of the Euclidean action in functional space. It is convenient to write the prefactor $\mathcal{P}$ as a product of the bubble's growth rate and a factor proportional to the ratio of the determinant of the fluctuation operator around the bubble configuration relative to that around the homogeneous metastable state [12]. For the relativistic case, in the thin-wall limit, we have [13]:

$$
\mathcal{P}=\frac{16}{3 \pi}\left(\frac{\sigma}{3 T}\right)^{3 / 2} \frac{\sigma \eta R_{c}}{\xi^{4}(\Delta \omega)^{2}} .
$$

Here, $\eta$ and $\xi$ are respectively the shear viscosity and the correlation length in the symmetric phase and $\Delta \omega$ is the enthalpy density difference between the two phases. $\sigma$ is the surface tension of the interface, which is related to the critical radius and the difference in pressure between the metastable phase and the true vacuum, $\Delta p$, as $R_{c}=2 \sigma / \Delta p$. The thin-wall limit is a good approximation in the limit of small supercooling, $\theta=1-T / T_{c} \ll 1$. In this limit, the decay rate can be written as

$$
\Gamma=\mathcal{P} \exp \left(-\frac{4 \pi \sigma}{3 T} R_{c}^{2}\right)=\mathcal{P} \exp \left(-\frac{16 \pi}{3} \frac{\sigma^{3}}{T_{c} \ell^{2} \theta^{2}}\right),
$$

where $\ell$ is the latent heat density. For increasing values of the supercooling, $\theta$, the argument of the exponential decreases and the decay rate increases. Equivalently, the critical radius becomes smaller, so that it is easier to nucleate supercritical bubbles. The only way in which the finite size of the system can affect the decay rate is through its influence on the supercooling.

Before considering the effects of the finite size of the system on the nucleation process, let's briefly examine the 
definition of metastable states in equilibrium statistical mechanics. This requires a coarse graining procedure which needs be implemented as follows [14]. The system of size $L$ is divided into cells centered at positions $\vec{x}$. The cell size, or the "coarse graining length" $\lambda_{c g}$, should be appreciably larger than the underlying lattice spacing $a$. Moreover, within each cell, the relevant order parameter, $\phi$, should vary smoothly in space and the equilibration time of the system should be much faster than the processes under consideration. By construction, the coarse-grained free energy will depend on the scale $\lambda_{c g}$. If $\lambda_{c g}$ is small enough as compared to the correlation length, $\xi$, phase separation within a cell cannot occur and one can define a coarse-grained free energy, $F[\phi]$, such as the one we considered before. The condition $\lambda_{c g}<<\xi$ therefore represents the physically motivated restriction to the partition function that allows for a welldefined coarse-grained description of metastable states. As a corollary, increasing the value of the coarse-graining length relative to the correlation length would incorporate more and more fluctuation modes, which were excluded by this ultraviolet cut-off. The free energy $F$ would then flatten out completely in the limit $\lambda_{c g} / \xi \rightarrow \infty$ approaching the equilibrium description with no metastable branch. In summary, for a system to be characterized by a well-defined LandauGinzburg coarse-grained free energy one requires the following clean separation of scales: $a<<\lambda_{c g}<<\xi<<L$.

We now return to finite size effects on nucleation. The only effect that might be relevant to the nucleation rate, $\Gamma$, is the rounding of singularities, since they will affect the degree of supercooling. The relevant physical quantity can be calculated in a finite-size scaling framework, resulting in the following expression [15]:

$$
\frac{\Delta T_{\text {round }}}{T_{c}} \approx \frac{2 T_{c}}{\ell L^{d}}
$$

where $L$ is the typical length scale of the system and $d$ is the number of dimensions. The quantity $\Delta T_{\text {round }}$ is a measure of the smoothening of singularities due to the finite size of the system. (A similar result is obtained in model studies of the equation of state of a finite quark gluon plasma [16].) It corresponds to the (now non-vanishing) width in temperature of the region where the energy density of the system suffers an abrupt jump, around $T=T_{c}$, which is itself shifted. This phenomenon enters the decay rate as a minimum value for $\theta$ which reflects the uncertainty in the supercooling due to the finite size of the system.

The procedure to derive (3) assumes the co-existence of two phases + and - at and near a first order phase transition and Eq. (3) follows from expanding the free energies of the two phases around $T_{c}$. (Implicit in the derivation is the requirement that the free energies of the two phases are very close to each other - i.e., the system is minimally supercooled.) In fact, it is the requirement that the two states contribute to the average energy with equal weight that determines $T_{c}(L)$ - the effective critical temperature for a system of size $L . \Delta T=T_{c}(L)-T_{c}(\infty)$ refers to the fact that the critical temperature cannot be resolved on a scale smaller than $\Delta T$ relative to $T_{c}(\infty)$. The supercooling parameter that appears in the nucleation rate is defined to be
$\theta=\left(T_{c}-T\right) / T_{c}$ (with $\left.T_{c}=T_{c}(\infty)\right)$. For a finite system, $T$ could not be closer to $T_{c}(\infty)$ than $T_{c}(L)$. It therefore makes little sense to talk about supercooling for temperatures less than this difference. On the contrary, there is no problem resolving differences larger than $\Delta T$ - hence its identification as a lower bound on cooling. This conclusion should be contrasted with the arguments presented in Ref. [17].

Although our discussion is restricted to finite size effects on first order transitions, we note that the case of a second order transition is also discussed in Ref. [15]. The effect of finite sizes in a second order phase transition is qualitatively different from that in a first order phase transition. The reason is likely that in a second order transition, the rounding is due to the correlation length being limited by the lattice size. In Ref. [15], for instance, one defines a quantity $V_{L}$ (which is related to physical quantities) and demonstrates that this quantity has a minimum in first order transitions for a range of lattice sizes. The same quantity, in a system with a second order transition, has a minimum that disappears very rapidly with change in lattice size. Thus a simple extrapolation of Eq. (3) to a second order transition would not be correct.

For phase transitions in the early universe, $L$ is given by the radius of the universe at a given time (or temperature). Going back to very early times, $t \sim 10^{-4} \mathrm{~s}$ and $T \sim 10^{12} \mathrm{~K} \sim 10^{-1} \mathrm{GeV}$, we can use the elementary particle model [18] to obtain an approximate equation of state: $3 p \approx \epsilon \approx \pi^{2} N(T) T^{4} / 30$, where $N(T)=N_{\text {bosons }}(T)+$ $(7 / 8) N_{\text {fermions }}(T)$ is the total number of degrees of freedom, $\epsilon$ is the energy density and $p$ is the pressure. From Einstein's field equations, one can derive the relation between time and energy density in the early universe, which links the age of the universe and its temperature in the following way

$$
t \approx\left(\frac{3}{32 \pi G \epsilon}\right)^{1 / 2} \approx \frac{1}{4 \pi}\left(\frac{45}{\pi N(T)}\right)^{1 / 2} \frac{M_{P l}}{T^{2}}
$$

where $M_{P l}$ is the Planck mass and $G$ is Newton's gravitational constant. The radius of the universe, as given by the particle horizon in a Robertson-Walker spacetime [19], $d_{h}(t)=t /(1-n)$, where the scale factor grows as $a(t) \sim t^{2 / 3(1+w)}=t^{n}$ and $p=w \epsilon$, has the following form:

$$
L_{\text {univ }}(T) \approx \frac{1}{4 \pi}\left(\frac{1}{1-n}\right)\left(\frac{45}{\pi N(T)}\right)^{1 / 2} \frac{M_{P l}}{T^{2}}
$$

For the assumed equation of state, $w=1 / 3$ and $n=1 / 2$. If we enter temperature in $\mathrm{GeV}$, the typical length scale of the early universe is given, in fermi units, by $L_{\text {univ }}(T) \approx$ $\mathcal{A} / T^{2} \sqrt{N(T)}$, where $\mathcal{A}=1.45 \times 10^{18}$.

It is clear that, due to the large factor $\sim 10^{18}$ (coming from $\left.M_{P l}\right), L_{u n i v}$ will be of importance for supercooling only for extremely high values of the temperature. Since $N(T) \approx 50$ for the cases of the QCD and the electroweak phase transitions, one would need $T \sim 10^{8} \mathrm{GeV}$ to be subject to appreciable finite-size effects on the nucleation rate. Therefore, since $T_{c}^{Q C D} \sim \mathcal{O}\left(10^{-1}\right) \mathrm{GeV}$ and $T_{c}^{E W} \sim \mathcal{O}\left(10^{2}\right) \mathrm{GeV}$, these effects are negligible in such cases. They might prove to be relevant in the case 
of GUT's transitions, where the critical temperature will be much higher.

For the first order transition of a quark-gluon plasma into a hadronic gas in a high-energy heavy ion collision, one can easily estimate the role of finite-size effects on the nucleation rate. The parameters entering Eq. (3) are approximately given by $T_{c} \approx 150 \mathrm{MeV}$ [20], $\ell \approx 4 B \approx 4 \times(150$ $\mathrm{MeV})^{4}$, where $B$ is the bag constant, and $L \approx 10 \mathrm{fm}$. The minimal amount of supercooling undergone by the plasma is then negligible, of the order of $10^{-3}$. (For more conservative, smaller values of $L$, the minimum value of $\theta$ is still very small.) The system can in principle probe the entire domain in $\theta$, and there are no constraints on homogeneous nucleation from this side. The question of how fast the system probes the nucleation region, before reaching the spinodal regime, as compared to the nucleation rate was discussed in Ref. [11, 21, 22]. Recently, it has been speculated that the RHIC data suggest an explosive hadron production due to a rapid variation of the effective potential for QCD close to $T_{c}$. The theoretical reasoning is based on the results of the Polyakov Loop Model [23] for the deconfining phase transition, which lead to a very fast spinodal decomposition regime [24].

After the nucleation of a given supercritical bubble, it will grow with a certain velocity. The set of all supercritical bubbles created integrated over time will eventually drive the complete phase conversion in a finite system. The scales that determine the importance of finite-size effects are the typical linear size of the system, the radius of the critical bubble and the correlation length. For the reasons discussed above, the case of cosmological phase transitions is to an excellent approximation free from finite-size corrections. The enormous numerical value of the Planck mass washes out every other scale. Therefore, in what follows we will address the quark-gluon-hadron phase transition in heavy ion collisions.

For definiteness, let us assume our system is characterized by a coarse-grained free energy of the form

$$
F(\phi, T)=\int d^{d} x\left[\frac{1}{2}(\nabla \phi)^{2}+U(\phi, T)\right],
$$

where $U(\phi, T)$ is some Landau-Ginzburg potential whose coefficients depend on the temperature, and $\phi(\vec{x}, t)$ is a scalar field. For the cases to be considered in this paper, the order parameter, $\phi$, is not a conserved quantity, and its evolution is given by the time-dependent Landau-Ginzburg equation [1]

$$
\frac{\partial \phi}{\partial t}=-\gamma \frac{\delta F}{\delta \phi}=\gamma\left[\nabla^{2} \phi-U^{\prime}(\phi, T)\right],
$$

where $\gamma$ is the response coefficient which defines a time scale for the system. Eq. (7) is a standard reaction-diffusion equation, and describes the approach to equilibrium [25].

If $U(\phi, T)$ is such that it allows for the existence of bubble solutions (taken to be spherical for simplicity), then supercritical (subcritical) bubbles expand (shrink), in the thinwall limit, with the following velocity:

$$
\frac{d R}{d t}=\gamma(d-1)\left[\frac{1}{R_{c}}-\frac{1}{R(t)}\right],
$$

where $R_{c}=(d-1) \sigma / \Delta F$ and $\Delta F$ is the difference in free energy between the two phases. Eq. (8) is an example of the Allen-Cahn equation [1], which relates the velocity of a domain wall to the local curvature. The response coefficient, $\gamma$, can be related to some characteristic collision time as will be done later.

The description of the late-stage domain coarsening is given by the Kolmogorov-Avrami theory [1], which contains the following assumptions: (i) bubbles grow without substantial deformation and are uncorrelated; (ii) the nucleation rate is a constant; (iii) the bubble growth velocity is constant, $v=d R / d t=\gamma(d-1) / R_{c}$ (limit $R \rightarrow \infty$ ). The quantity which is usually computed is the volume fraction of the stable phase.

However, one can measure the importance of finite-size effects for the case of heavy-ion collisions by comparing, for instance, the asymptotic growth velocity $\left(R>>R_{c}\right)$ for nucleated hadronic bubbles to the expansion velocity of the plasma. In the Bjorken picture, one assumes that the central rapidity region exhibits longitudinal expansion, so that $z(t)=v_{z} t$, where $v_{z}$ is the collective fluid velocity. Conservation of total entropy leads to adiabatic expansion and the following cooling behavior: $\left(t / t_{i}\right)=\left(T_{i} / T\right)^{3}$. The typical length scale of the expanding system is then

$$
L(T) \approx\left(v_{z} t_{c}\right)\left(\frac{T_{c}}{T}\right)^{3}=L_{0}\left(\frac{T_{c}}{T}\right)^{3},
$$

where $L_{0} \equiv L\left(T_{c}\right)$ is the initial linear scale of the system for the nucleation process which starts at $T \leq T_{c}$.

The relation between time and temperature provided by the cooling law that emerges from the Bjorken picture suggests the comparison between the following "velocities":

$$
v_{b} \equiv \frac{d R}{d T}=-\left(\frac{3 b \ell L_{0}}{2 v_{z} \sigma T_{c}^{2}}\right)\left(\frac{T_{c}}{T}\right)^{5}\left(1-\frac{T}{T_{c}}\right)
$$

the asymptotic bubble growth "velocity", and the plasma expansion "velocity"

$$
v_{L} \equiv \frac{d L}{d T}=-\frac{3 L_{0}}{T_{c}}\left(\frac{T_{c}}{T}\right)^{4}
$$

The quantity $b$ is a number of order one to first approximation, and comes about in the estimate of the phenomenological response coefficient $\gamma(T) \approx b / 2 T$ (see Ref. [26] for details).

Using the numerical values adopted previously and $\sigma / T_{c}^{3} \sim 0.1$, we obtain

$$
\frac{v_{b}}{v_{L}} \approx \frac{20}{v_{z}}\left(\frac{T_{c}}{T}-1\right)
$$

One thus observes that the bubble growth velocity becomes larger than the expansion velocity for a supercooling of or$\operatorname{der} \theta \approx v_{z} / 20 \leq 5 \%$. A simple estimate points to a critical radius larger than $1 \mathrm{fm}$ at such values of supercooling (see 
also [11]). Therefore, finite-size effects appear to be an important ingredient in the phase conversion process right from the start in the case of high-energy heavy-ion collisions.

Although the arguments presented here are based solely on analytic considerations and simple estimates, one can draw several conclusions. It is clear that the nucleation process in both the electroweak and the QCD cosmological phase transitions is almost insensitive to effects coming from the finite size of the universe in its early stages of evolution. On the other hand, in the case of heavy ion collisions the decay rate is just slightly affected by finite-size effects, whereas in the late-stage growth the separation of length scales seems to become small very early in the hadronization process. In order to address the question of hadronization after a first-order transition in a heavy ion collision picture, one should then perform finite-size real-time lattice simulations. In fact, lattice methods have been successfully applied to the study of homogeneous nucleation in different contexts $[2,27,28]$. One can thereby avoid the drawbacks implied by analytical approximations, such as the thin-wall hypothesis. Moreover, one could be able to control the behavior of domains and study, for instance, scaling properties. Results in this direction will be reported in a future publication [29]. It is also interesting to study the hydrodynamics of nuclear matter at chiral limit as a phenomenological description of the chiral transition in an expanding quark-gluon plasma [30-33]. In fact, the coupling between chiral and hydrodynamical modes seem to play a very non-trivial role in the dynamics of hadronization [33]

E.S.F. is partially supported by CAPES, CNPq, FAPERJ and FUJB/UFRJ. R.V. is supported by the U.S. Department of Energy under Contract No. DE-AC02-98CH10886 and by the RIKEN-BNL Research Center at BNL.

\section{References}

[1] J. D. Gunton, M. San Miguel, and P. S. Sahni in Phase Transitions and Critical Phenomena (Edited by C. Domb and J. L. Lebobowitz, Academic Press, London, 1983), vol. 8; K. Binder, Rep. Prog. Phys. 50, 783 (1987).

[2] P. A. Rikvold and B. M. Gorman in Annual Reviews of Computational Physics I (Edited by D. Stauffer, World Scientific, Singapore, 1994).

[3] E. S. Fraga and R. Venugopalan, arXiv:hep-ph/0304094.

[4] D. Boyanovsky, hep-ph/0102120.

[5] E.V. Shuryak, Phys. Rept. 61, 71 (1980); K. Kajantie and L. McLerran, Ann. Rev. Nucl. Part. Sci. 37, 293 (1987); B. Müller, Rept. Prog. Phys. 58, 611 (1995); J. Harris and B. Müller, Ann. Rev. Nucl. Part. Sci. 46, 71 (1996); S.A. Bass, M. Gyulassy, H. Stöcker, and W. Greiner, J. Phys. G25, R1 (1999).

[6] L. P. Csernai and J. I. Kapusta, Phys. Rev. Lett. 69, 737 (1992); J. I. Kapusta, A. P. Vischer, and R. Venugopalan, Phys. Rev. C 51, 901 (1995); J. I. Kapusta and A. P. Vischer, ibid. 52, 2725 (1995); E. E. Zabrodin, L. Bravina, H. Stocker, and W. Greiner, ibid. 59, 894 (1999); P. Shukla, A. K. Mohanty, S. K. Gupta, and M. Gleiser, ibid. 62, 054904 (2000).
[7] M. Trodden, Rev. Mod. Phys. 71, 1463 (1999).

[8] R. A. Malaney and G. J. Mathews, Phys. Rept. 229, 145 (1993).

[9] Proc. of Quark Matter 2002, Nucl. Phys. A 715, 1 (2003).

[10] O. Scavenius and A. Dumitru, Phys. Rev. Lett. 83, 4697 (1999).

[11] O. Scavenius, A. Dumitru, E. S. Fraga, J. T. Lenaghan, and A. D. Jackson, Phys. Rev. D 63, 116003 (2001).

[12] J. S. Langer, Ann. Phys. 41, 108 (1967); ibid. 54, 258 (1969); ibid. 65, 53 (1971); J. S. Langer and L. A. Turski, Phys. Rev. A 8, 3230 (1973); L. A. Turski and J. S. Langer, ibid. 22, 2189 (1980).

[13] L. P. Csernai and J. I. Kapusta, Phys. Rev. D 46, 1379 (1992); R. Venugopalan and A. P. Vischer, Phys. Rev. E 49, 5849 (1994).

[14] J. S. Langer, Physica 73, 61 (1974).

[15] K. Binder and D. P. Landau, Phys. Rev. B 30, 1477 (1984). M. S. S. Challa, D. P. Landau, and K. Binder, ibid. 34, 1841 (1986).

[16] C. Spieles, H. Stöcker, and C. Greiner, Phys. Rev. C 57, 908 (1998).

[17] B. Banerjee and R. V. Gavai, Phys. Lett. B 293, 157 (1992); Mod. Phys. Lett. A 11, 615 (1996).

[18] S. Weinberg, Gravitation and Cosmology (John Wiley \& Sons, 1972).

[19] E. W. Kolb, M. S. Turner, The Early Universe (Perseus, 1993).

[20] F. Karsch, Nucl. Phys. A 698, 199 (2002).

[21] E. E. Zabrodin, L. V. Bravina, L. P. Csernai, H. Stocker, and W. Greiner, Phys. Lett. B 423, 373 (1998).

[22] P. Shukla and A. K. Mohanty, Phys. Rev. C 64, 054910 (2001).

[23] R. D. Pisarski, Phys. Rev. D 62, 111501 (2000).

[24] O. Scavenius, A. Dumitru, and A. D. Jackson, Phys. Rev. Lett. 87, 182302 (2001).

[25] In order to include the effect of fluctuations, one should add a Langevin noise term in (7).

[26] K. Kajantie, Phys. Lett. B 285, 331 (1992).

[27] S. Gupta, Phys. Lett. B 325, 418 (1994).

[28] G. D. Moore and K. Rummukainen, Phys. Rev. D 63, 045002 (2001). G. D. Moore, K. Rummukainen, and A. Tranberg, JHEP 0104, 017 (2001).

[29] E. S. Fraga, A. Krasnitz, and R. Venugopalan, to appear.

[30] D. T. Son, Phys. Rev. Lett. 84, 3771 (2000).

[31] I. N. Mishustin and O. Scavenius, Phys. Rev. Lett. 83, 3134 (1999).

[32] K. Paech, H. Stöcker, and A. Dumitru, nucl-th/0302013.

[33] C. E. Aguiar, E. S. Fraga, and T. Kodama, arXiv:nuclth/0306041. 